\title{
A review of the research on music therapy in hospice care ${ }^{\dagger}$
}

Review

\author{
Meng-Xue Fua, ${ }^{a}$, Qin Lin ${ }^{b}$, Ji-Jun Wuc , Xiao-Li Zhong ${ }^{d}$, Ling-Na Yange, Guo-Jing Han ${ }^{f}$ \\ ${ }^{a}$ Department of Nursing, People's Hospital of Jianyang City, Jianyang, Sichuan 641400, China \\ ${ }^{b}$ Department of Nursing, Sichuan Nursing Vocational College, Chengdu, Sichuan 610100, China \\ 'Department of Nursing, People's Hospital of Deyang City, Deyang, Sichuan 618000, China \\ ${ }^{d}$ Department of Nursing, Panzhihua University, Panzhihua, Sichuan 617000, China \\ eDepartment of Scientific Research, Sichuan Nursing Vocational College, Chengdu, Sichuan 610100, China \\ ${ }^{t}$ Department of Pediatrics, Sichuan Provincial Hospital for Women and Children, Chengdu, Sichuan 610041, China
}

Received: 19 December 2019; Accepted: 17 January 2020; Published: 20 September 2020

Abstract: Objective: To summarize the research progress of music therapy in hospice care and to provide reference for further research Methods: A wide range of literatures on music therapy and hospice care at home and abroad were searched and read, and the literature was integrated, judged, analyzed, and summarized.

Results: The related research on music therapy in hospice care mainly focused on western developed countries such as Europe and the United States. China is still in the stage of theoretical exploration, lacking qualitative research based on case investigation and empirical research based on data statistics.

Conclusions: Music therapy supports the management of symptoms in hospice care, which can meet the diverse needs of patients and their family members including physical, psychological, social, and spiritual.

Keywords: music therapy • hospice care $\bullet$ application and mechanism • research progress $\bullet$ review

(c) Shanxi Medical Periodical Press.

\section{Introduction}

In the new era of medical reform in China, hospice care is a latest way to cope with the shortage of medical resources, the aging of the population, and the increasing number of cancer patients. In recent years, music therapy has been increasingly used as an adjunct to support symptom management in hospice care. Music therapy has been well developed in western developed countries such as those in Europe and the United States. China's research on music therapy in hospice care is still in its infancy. Consistent with the goal of hospice care, the main purpose of music therapy is to improve the quality of life by solving the psychological needs of patients and promoting communication. ${ }^{1}$ It can be seen that the study of music therapy for patients during hospice care is necessary and supporting the implementation of the hospice plan is a very important part.

† This project was supported by Construction and Empirical Study of Competency Index System for Nurses Specializing in Hospice Care and Nursing (No. 19PJ042)

How to cite this article: Fu MX, Lin Q, Wu JJ, Zhong XL, Yang LN, Han GJ. A review of the research on music therapy in hospice care. Front Nurs. 2020;3:179-184. 
Therefore, this paper aims to summarize the research progress of music therapy in hospice care, and to explore the application, mechanism, and advantages of music therapy in hospice care, in order to further enrich and improve the content of music therapy in hospice care and provide reference.

\section{The concept and content of hospice care}

Hospice care is also known as NingYang care, terminal care, or end of life care. The hospice care originated in the United Kingdom in the 1960s. The term "hospital care" began in Taiwan, China. It means that both "medical" and "nursing" should be paid equal attention to, so that patients can get "tranquility." According to the World Health Organization's definition of hospice care, it refers to the provision of enthusiasm and full-hearted care for end-stage patients who are not responding to curative treatment, in order to maintain the best quality of life of patients and their families, mainly through pain control and alleviate other symptoms of discomfort on the body while dealing with psychological, social, and spiritual problems of patients and their families. The hospice care was initially targeted at cancer patients ${ }^{2}$ and gradually extended to other critically ill patients, such as people having AIDS and motor neuron atrophy, and those who are suffering from dementia and aging elders. The hospice care includes the whole person (body, mind, and spirit as a whole), the whole family (body, heart, grief, etc.), the whole process (care for the patient until the passing of life and the grief counseling of the family members after the death), and the whole team (complete professional team care for) four full care Four-care is the most important service connotation in the early days of hospice care. The hospice care is divided into three types: inpatient care, day care, and home care. The unrestricted nature of the implementation site greatly enriches its applicability and practicality.

\section{The concept and content of music therapy}

The method of music therapy originated in the ancient Greek period. With the establishment of the National Music Therapy Association in 1950, music therapy was bring into being an independent profession and in 1978 was introduced into the field of hospice care. Music therapy is defined as the use of sound and music to support and develop physical, mental, and social mental health in a growing relationship between clients/patients and therapists. ${ }^{3}$ Under the guidance of Roy adaptation model, music as a positive stimulating factor can improve the patient's ability to cope at the end of life, help to accept the symptom management of hospice patients, and improve their quality of life. ${ }^{4}$

Music therapy is mainly through the use of its various techniques; in the individual session (one-on-one treatment) of music therapy, patients can determine the frequency of treatment time (1-3 times a week) and duration (20-90 min), and music therapy techniques and content can be selected in each session. Music therapy is divided into three types: accepted music therapy, impromptu music therapy, and re-creation music therapy. Acceptable music therapy focuses on using live or recorded music, a feature that includes active listening. Nurses can help patients choose the music that evokes memories or feelings and help patients turn their attention to emotions and body. It is a technique that can be used even by nurses having no formal music training. Impromptu music therapy provides interactive and musical dialogs, and patients can perform as they change their emotional sensations, aiming to enhance their self-expression. Re-creation music therapy allows patients to not only listen to music, but more importantly, participate in music activities in person, aiming to train patients to enhance their reaction and interpersonal communication from music behavior. The specific measures that can be used for music therapy mainly include listening to images through music, song creation, lyric analysis, singing, improvisation, instrumental performance, and relaxation therapy to treat and care for patients and their family needs for therapeutic care. Music therapy often requires treatment and care in hospice care including social (isolation, loneliness, boredom, etc.), emotions (depression, anxiety, anger, fear, depression, etc.), cognition (neural damage, disorientation, confusion, etc.), the body (pain, shortness of breath, etc.), and the spirit (lack of spiritual connection, need for spiritualbased rituals, etc.).

\section{Application and mechanism of music therapy in hospice care}

\subsection{The application of music therapy in hospice care}

Music therapy can induce relaxation, reduce physical and emotional pain, provide spiritual support, promote the formation of intimacy and belonging, and provide 
opportunity for emotional expression. A large body of literature indicate that during hospice care, music can provide treatment and care to patients, ${ }^{5}$ and both patients and family members can benefit from music therapy. Marco Warth ${ }^{6}$ provides a new music therapy intervention called "Song of Life" (SOL) for terminally ill patients with cancer to assess the life status and mental health of those receiving hospice care. SOL specific measures include biographical interviews and lullaby-based methods for patients to perform live performances that are highly relevant. The results showed a moderate improvement in health status, relaxation, disease concerns, and pain. In a prospective case study conducted abroad, ${ }^{7}$ the use of Body Tambura's music therapy for the perception of palliative care, especially pain, was documented. Body Tambura is a new tool in the field of accepted music therapy inspired by classical Indian Tampura. It is designed to be placed and attached to the human body. The acoustic space of the harmonics generated by the sound of the instrument and the vibration of the body directly felt by the patient, thereby receiving music therapy. The results of this study show that when using Tambura to implement music therapy, both patients and implementers can share the positive experience of music therapy; not only the patient's body pain is significantly reduced, but also the hospice team and the patient's relatives can get music therapy. The music healing heritage can improve the bereavement of the mourners through contact with the deceased. Callaghan $^{8}$ creates opportunities for intimate contact with patients and/or family members with life-threatening conditions - based on music therapy to help patients create heritage, including tangible heritage (music and family members' musical creations) and intangibles heritage (the beautiful memories of the process of creating music for patients and family members). The results of the study showed that the song creation with the patient made the family members who lost their loved ones feel comfortable.

\subsection{Mechanism of music therapy in hospice care}

Music therapy consists of a variety of mechanisms, mainly in the four areas of support, emotion, spirit, and society, which can provide physical, psychological, emotional, and social support. Literature analysis is in the field of support, ${ }^{9}$ which can distract body and mind, through music therapy, allowing patients to shift focus from disease to music and other enjoyable things; it can help patients to rediscover their identity, from "patient", as someone with a unique past, interest, and personality. In the field of emotion, according to the literature, ${ }^{10}$ a key therapeutic mechanism of music therapy is that it can create a safe environment in which patients can express their emotions and expressions. Porter ${ }^{11}$ and others complement this conclusion in the qualitative study of the mechanisms of music therapy during hospice care, i.e., music therapy can help patients express themselves in ways they never thought of. While patients use music therapy to express themselves, they can also provide comfort to their loved ones. ${ }^{12,13}$ In the spiritual field, the key therapeutic mechanism identified in the literature,,$^{9,14,15}$ music therapy can help patients find meaning in life, providing patients with a mechanism to surpass their own situation and obtain peace. In the social sphere, a key therapeutic mechanism identified in the literature is that music therapy helps to strengthen the social connection between patients and their loved ones. ${ }^{16,17}$ Holistic care and good communication are important components of high-quality hospice care for patients and their families/caregivers, and music therapy can provide opportunities to enhance communication between patients and medical staff, and promote the formation of quality holistic care.

\section{Benefits and limitations of music therapy in hospice care}

Pain treatment for palliative care patients is challenging. Auxiliary methods of pain management are desirable, and music therapy offers non-pharmacological and safe alternatives. Numerous studies have shown that music therapy is beneficial for pain, dyspnea, physical comfort, and body perception. 18,19 The patient's mental needs can also be fully understood and respected, ${ }^{20}$ negative emotions such as anxiety, depression, irritability, and so on can be reduced, and the happiness index is significantly improved. A retrospective study showed, ${ }^{21}$ in a music therapy intervention for hospice patients, $96 \%$ of patients responded positively, patients' pain, anxiety, depression, shortness of breath, mood, facial expressions, and vocal scores improved significantly. In a mixed study, Peng $\mathrm{CS}^{22}$ conducted a live music intervention course for patients who were consulted for palliative care. In palliative care, compared to the previous equivalent time period after the implementation of music therapy, the use of opioids in patients was significantly reduced, with significant reductions in pain, anxiety, nausea, shortness of breath, and depression. Family members play an important role in hospice care, and hence family satisfaction is also seen as an important part of highquality hospice care. In a survey of family members 
who received music therapy, $82 \%$ of family members said their self-stress levels, mood, and quality of life improved, and $49 \%$ of family members suggested that further music therapy was necessary for patients. The unique quality of music helps to establish a trusting therapeutic relationship. Its flexibility and adaptability enable medical workers to work intensively even in a very short period of time to meet the overall needs of patients and their family members. ${ }^{23}$

In summary, during hospice care, music therapy has been proven to relieve the physical and emotional suffering of the patients, and it can be used in a variety of environments, is economical, and is environmentally friendly. However, at present, nursing staff in China have limited knowledge and skills in using music as therapy, and lack the resources and ability to implement it. Therefore, professional training is needed for active implementation of music therapy.

\section{References}

1. McConnell T, Graham-Wisener L, Regan J, et al. Evaluation of the effectiveness of music therapy in improving the quality of life of palliative care patients: a randomised controlled pilot and feasibility study. Pilot Feasibility Stud. 2016;2:70.

2. McClean S, Bunt L, Daykin N. The healing and spiritual properties of music therapy at a cancer care center. Altern Compl Med. 2012;18:402-407.

3. Bunt L, Hoskyns S. The Handbook of Music Therapy. Hove: Brunner Routledge; 2002:11.

4. Bowers TA, Wetsel MA. Utilization of music therapy in palliative and hospice care: an integrative review. J Hosp Palliat Nurs. 2014;16:231-239.

5. Broadhurst D, Islam Z. P-7 "My story in music" at loros hospice: the story so far. Support Palliat Care. 2015;5:A3.1-A3.

6. Warth M, Kessler J, Kampen JV, et al. "Song of Life": music therapy in terminally ill patients with cancer. Support Palliat Care. 2018;8:bmjspcare-2017-001475.

7. Dietrich C, Teut M, Lopoyetum S K, et al. Treating palliative care patients with pain with the Body Tambura: a prospective case study at St Joseph's hospice for the dying destitute in Dindigul South India. Eur J Integr Med. 2015;7:33-34.

8. Callaghan C. Music therapy preloss care though legacy creation. Prog Palliat Care. 2013;21:78-82.

9. McConnell T, Porter S. Music therapy for palliative care: a realist review. Palliat Support Care. 2017;15:454-464

\section{Conclusions and outlook}

The research of music therapy in hospice care is mainly concentrated in western developed countries such as Europe and America, whereas China is still in the stage of theoretical exploration. With the application of music therapy in the field of hospice care becoming a trend, qualitative research based on case study and empirical research based on data statistics are urgently needed in China.

\section{Ethical approval}

Ethical issues are not involved in this paper.

\section{Conflicts of interest}

All contributing authors declare no conflicts of interest.
10. Heath B, Lings J. Creative songwriting in therapy at the end of life and in bereavement. Mortality. 2012;17:106-118.

11. Porter S, Mcconnell T, Clarke M, et al. A critical realist evaluation of a music therapy intervention in palliative care. BMC Palliat Care. 2017;16:70.

12. Dimaio L. Music therapy entrainment: a humanistic music therapist's perspective of using music therapy entrainment with hospice clients experiencing pain. Music Ther Perspect. 2010;28:106-115.

13. Sato Y. Musical life review in hospice. Music Ther Perspect. 2011;29:31-38.

14. McClean S, Bunt L, Daykin N. The healing and spiritual properties of music therapy at a cancer care centre. J Altern Complement Med. 2012;18:402-407.

15. Sato Y. Musical life review in hospice. Music Ther Perspect. 2011;29:31-38.

16. O'Kelly J, Koffman J. Multi-disciplinary perspectives of music therapy in adult palliative care. Palliat Med. 2007;21:235-243.

17. Leow QHM, Drury VB, Poon WH. A qualitative exploration of patients' experiences of music therapy in an inpatient hospice in Singapore. Int $J$ Palliat Nurs. 2010;16:344-350.

18. Teut M, Dietrich C, Deutz B, et al. Perceived outcomes of music therapy with Body Tambura in end of life care - a qualitative pilot study. BMC Palliat Care. 2014;13:18.

19. Polt G, Fink M, Schieder $H$, et al. Influence of music on the quality of life of palliative 
cancer patients. Wien Med Wochenschr. 2014;164: 179-183.

20. Burns DS, Perkins SM, Tong Y, et al. Music therapy is associated with family perception of more spiritual support and decreased breathing problems in cancer patients receiving hospice care. Pain Symptom Manage, 2015;50:225-231.

21. Gallagher LM, Lagman R, Rybicki L. Outcomes of music therapy interventions on symptom management in palliative medicine patients. Am J Hosp Palliat Care. 2017;35:1049909117696723.

22. Peng CS, Baxter K, Lally KM. Music intervention as a tool in improving patient experience in palliative care. Am J Hosp Palliat Care. 2019;36:45-49.

23. Gallagher LM, Lagman R, Bates D, et al. Perceptions of family members of palliative medicine and hospice patients who experienced music therapy. Support Care Cancer. 2017;25:1769-1778. 